九州大学学術情報リポジトリ

Kyushu University Institutional Repository

\title{
Evolving Color Paint
}

Mizutani, Eiji

Informations Systems Dept., Kansai Paint Co., Ltd.

\section{高木，英行}

Department of Acoustic Design, Kyushu Institute of Design

David M. Auslander

Department of Mechanical Engineering, University of California at Berkeley

http://hdl. handle. net/2324/4485873

出版情報: Proceedings of 1995 IEEE International Conference on Evolutionary Computation. 2, pp. 533-538, 1995-11-29. IEEE

バージョン :

権利関係 : 


\title{
Evolving Color Paint
}

\author{
Eiji Mizutani ${ }^{1}$, Hideyuki Takagi ${ }^{2}$, and David M. Auslander ${ }^{3}$
}

eiji@joho.kansai.co.jp, takagi@kyushu-id.ac.jp, dma@euler.berkeley.edu

1) Informations Systems Dept., Kansai Paint Co.,Ltd, 4-3-6 Fushimi Chuo, Osaka 541, Japan

2) Acoustic Design Dept., Kyushu Institute of Design, 4-9-1 Shiobaru, Minami-ku Fukuoka 815, Japan

3) Dept.of Mechanical Engineering, University of California at Berkeley, CA 94720, USA

\begin{abstract}
This paper presents an evolutionary computing intelligence for the paint industry. It integrates three principal components of soft-computing and a problem-specific knowledge to perform a computerized color recipe prediction; that is, neural networks (NNs), a fuzzy system (FS), and a genetic algorithm (GA) with a knowledge base (KB) complement each other in obtaining precise prediction outputs through the simulation of color paint manufacturing process.

In light of both potential versatility and practical validity in this industrial application, we have realized that the proposed hybrid system can yield advantages over other approaches in that it can evolve their results.
\end{abstract}

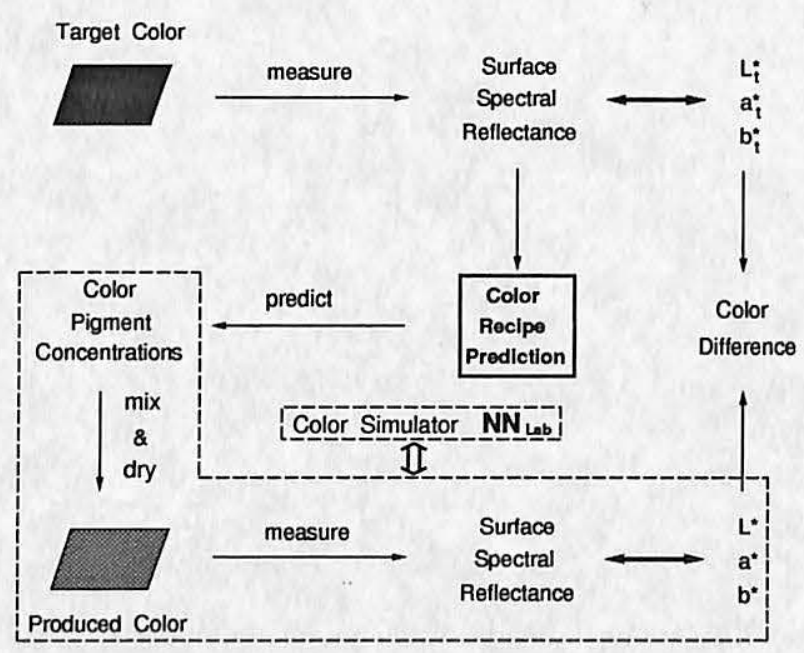

Fig. 1: Color paint manufacturing process.

\section{Introduction}

A color recipe prediction task is to predict which color pigments and what concentrations of the pigments are needed to produce the same color as a given reference color (see Figure 1). It is very difficult even for professional colorists to do well, yet human color perception is very sensitive and therefore the color matching must be done very well to meet acceptable standards. Since the widely-used

The authors would like to thank Mr. Kenichi Nishio (Sony Corp.), Dr. J.-S. Roger Jang (MathWorks, Inc.), and Mr. Tohru Hirayama (Kansai Paint Co.,Ltd) for their valuable advice. This work was mainly conducted when author ${ }^{1}$ was a graduate student at the University of California at Berkeley.
Kubelka-Munk theory requires some assumptions which limit the situations where the theory may be applied [10], simple NN approaches, just like an NN in Figure 3 (A), have been introduced to overcome the practical obstacles in this problem area $[2,9]$. The major concerns are (1) how to keep the practical number of necessary pigments, (2) how to consider the magnitude of the perceived color difference in the human visual sense, and (3) how to obtain precise pigment concentrations with enough precision to specify levels such as $0.01 \%$. The first concern is a kind of combinational problem for which the GA may be a good choice. We use NNs to cope with the second critical problem. In regard to the third concern, we use a fusion technique of NNs, an FS, and a GA with a KB, to evolve color pigment concentration vectors. We shall clarify the evolutionary mechanism in the subsequent sections.

\section{Hybrid System Architecture}

In the initial stage, starting points for a GA search are set by a fuzzy population generator and a multi-elite generator using results from NN approaches. In the evolutionary stage, the hybrid system tries to improve those results, which are already within some range of the ideal pigment concentrations. Two different NNs and a KB are used to make up the fitness function. Genes' pigment concentrations are passed to three functions which calculate fitness values individually. The three values are combined into the final fitness value. This mechanism is shown in Figure 2. 


\section{Initial Stage}

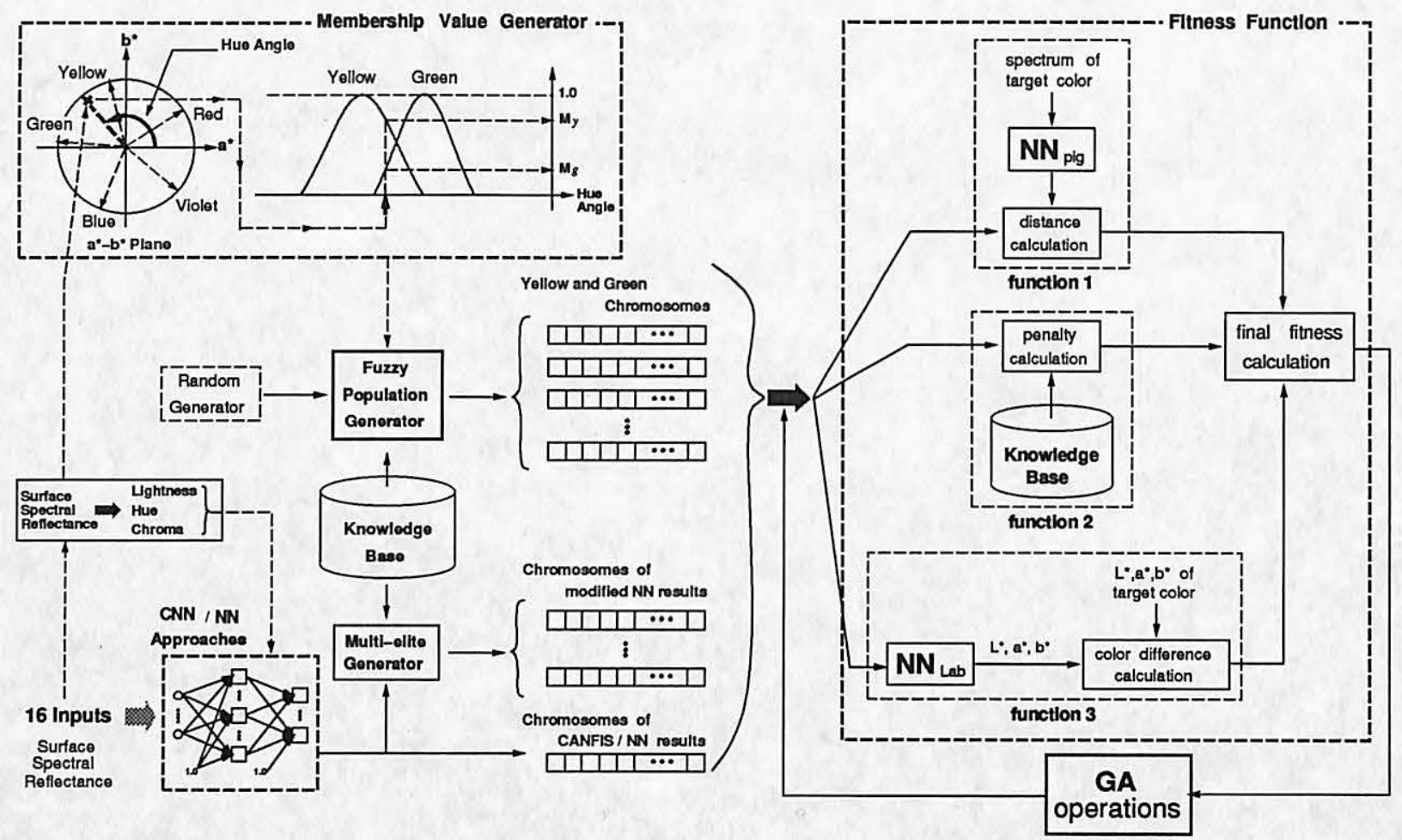

Fig. 2: Computational intelligence for color recipe prediction.

\subsection{Knowledge Base}

Knowledge may be useful in reinforcing some favorable aspects of genetic searches [6]. Performing the color recipe prediction task requires special knowledge; we believe that a $\mathrm{KB}$ plays an important role in helping the system evolve to recognize specific features of a target color. The KB has the following main rules:

Rule 1: Keep total proportions of color pigments around $100 \%$,

Rule 2: Keep the number of necessary color pigments around the ideal number,

Rule 3: Avoid use of complementary color pigments: e.g., Red and Green,

Rule 4: Avoid use of the same kind of color pigments at the same time.

We have 10 color pigments (10 outputs), including 3 pairs of the same kind of colorants: green, yellow, and red ones (see Figure 3 ). Note that $\operatorname{Red}_{1}$ and $\operatorname{Red}_{2}$, for example, have different characteristics. In this prediction task, the $100 \%$ rule (Rule 1) was emphasized in [9], also.

\subsection{Multi-elites Generator}

Firstly, the results of NN approaches are encoded into initial population as elite members. Then, a multi-elite generator produces more elites by modifying those results according to Rule 4 in the KB. Namely, the concentrations of the same color pig- ments are summed into one or another of them: e.g., $\operatorname{Red}_{1}+\operatorname{Red}_{2} \Rightarrow \operatorname{Red}_{1}$, or $\operatorname{Red}_{1}+\operatorname{Red}_{2} \Rightarrow$ $\operatorname{Red}_{2}$. This is derived from the fact that the simple NN tends to specify use of more than six pigments, though the desired number of pigments to produce any color in our data sets is fewer than five. Again, it is important to keep the number of pigments used at a practical level. Those multiple elite pigment vectors offer several different starting points for GA searches. The combination of several solutions may be effective in finding the optimal solution [5]. The other members are initialized by a fuzzy population generator. This seeding procedure is shown in Figure 2 (left).

\subsection{Fuzzy Population Generator}

The idea is to generate the initial population according to a fuzzy classification of a target color, which serves to determine color selection. First, we classify the target color into one of five color categories (red, yellow, green, blue, and violet) on the $\mathrm{a}^{*}-\mathrm{b}^{*}$ plane, which shows hue and chroma [10] (see also Section 2.4.3), and decide to what extent the desired color belongs to each color category using fuzzy membership functions $[7,4]$. We then generate initial color chromosomes by modifying randomly-generated chromosomes according to the rules in the KB. For example, when a target color 
looks greenish yellow, green chromosomes and yellow ones are generated; Green chromosomes have zero values in either Green $_{1}$ or Green f $_{2}$ pigment concentration and in red pigment concentrations because of the red-green complementary color relationship. It is effective to inactivate some genes which have information on the same color and a complementary color in order to eliminate redundant pigments at the initial stage.

The number of green chromosomes $\left(N u m_{\text {Green }}\right)$ and that of yellow ones $\left(\mathrm{Num}_{Y \text { ellow }}\right)$ are decided according to the following calculations:

$$
\begin{aligned}
\text { Pop rest }_{\text {retal }}-\text { Pop }_{N N}, & \text { Pop }_{\text {tot }} \\
\text { Num }_{\text {Green }} & =\frac{M_{g} \cdot P_{o p_{\text {rest }}}}{M_{y}+M_{g}}, \\
\text { Num } & =\frac{M_{y} \cdot P_{\text {ellow }}}{M_{y}+M_{g}}
\end{aligned}
$$

where two membership values, $M_{y}$ and $M_{g}$, show to what extent the target color belongs to the yellow category and the green one, respectively. Pop total denotes the total population number, and $P o p_{N N}$ means the number of elite chromosomes from the NN results including the chromosomes generated by the multi-elite generator.

\subsection{Fitness Function}

The fitness function consists of three functions; two neural fitness functions, and the KB-based fitness function.

\subsubsection{Function 1}

Using $N N_{\text {pig }}$, the first function evaluates genes' pigment concentration vectors according to the specified use of pigments. The $N N_{p i g}(16 \times 18 \times 21 \times 10$ neurons) maps surface spectral reflectance to a list of required pigments (see Figure $3(\mathrm{C})$ ). It gives just ON/OFF values to each output unit to predict which pigments should be used to produce the same color as the target color, where ON means "needed" pigment and OFF means "not needed." Table 1 shows the capability of this trained $N N_{\text {pig }}$. Function 1 evaluates each chromosome by calculating a distance in binary space (ON/OFF) after each chromosome's representation has been transformed into the ON/OFF format.

\subsubsection{Function 2}

The second function calculates a fitness value based on the KB described in Section 2.1. The fitness value depends on the extent to which genes' pigment concentration vector obeys the rules in the KB. To keep the GA search moving in a consistent direction, the $\mathrm{KB}$ is used in both the initial stage and the calculation of fitness values as illustrated in Figure 2.
Table: 1: Capabilities of different NN approaches in specifying necessary pigments. CNN is a neuro-fuzzy model. $N N_{1}$ is a simple $N N$, and $N N_{2}$ is an improved $N N_{1}$; they appear in Figure $3(\mathrm{~A}) . N N_{p i g}$ is a special $N N$ to predict necessary pigments as appeared in Figure $3(\mathrm{C})$.

\begin{tabular}{|l|c|c|c|c|}
\hline & $N N_{1}$ & $N N_{2}$ & CNN & $N N_{\text {pig }}$ \\
\hline $\begin{array}{l}\text { \# of unmatched patterns } \\
\text { in 302 test patterns }\end{array}$ & 299 & 74 & 73 & 27 \\
\hline $\begin{array}{l}\text { \# of unmatched units } \\
\text { in 3,020 output units }\end{array}$ & 911 & 106 & 98 & 48 \\
\hline $\begin{array}{l}\text { Predicted ave. \# of } \\
\text { required pigments to use }\end{array}$ & 6.66 & 3.90 & 3.89 & 3.96 \\
\hline
\end{tabular}

Table: 2: Average color difference predicted by $N N_{L a b}$ using 302 checking data. The inputs of the $N N_{L a b}$ were ideal pigment concentration vectors, and the results from three NN approaches; CNN is a neuro-fuzzy model, $N N_{1}$ is a simple $\mathrm{NN}$, and $N N_{2}$ is an improved $N N_{1}$. This shows potential capability of the $N N_{L a b}$.

\begin{tabular}{|c||c|c|c|c|}
\hline pigment vectors & ideal & $\mathrm{CNN}$ & $N N_{2}$ & $N N_{1}$ \\
\hline color difference & 0.567 & 1.976 & 2.847 & 5.921 \\
\hline
\end{tabular}

\subsubsection{Function 3}

The third function, based on $N N_{L a b}$, generates a fitness value in terms of color difference between a target color and each member's color whose pigment concentrations are predicted by the system. Because it is time-consuming to manufacture an actual color by mixing genes' specified values (see Figure 1), the $N N_{L a b}$ plays a crucial role as a color simulator to predict what color will be produced. The $N N_{L a b}(10 \times 11 \times 14 \times 3$ neurons $)$ maps color pigment concentrations to $L^{*}, a^{*}$, and $b^{*}$ (see Figure $3(\mathrm{~B})$ ). By plugging each member's pigment concentrations into $N N_{L a b}$, we can obtain predicted $L^{*}, a^{*}$, and $b^{*}$, to calculate the color difference between a target color and an individual color.

We adopted CIE $1976\left(L^{*}, a^{*}, b^{*}\right)$-space [10]. This defines the color difference and perceptual attributes of color, which are lightness, hue, and chroma as shown below:

$$
\begin{array}{ll}
\text { Color Difference } & \sqrt{\left(L_{t}^{*}-L^{*}\right)^{2}+\left(a_{t}^{*}-a^{*}\right)^{2}+\left(b_{t}^{*}-b^{*}\right)^{2}} \\
\text { Lightness } & L^{*} \\
\text { Hue } & \arctan \left(b^{*} / a^{*}\right) \\
\text { Chroma } & \sqrt{\left(a^{*}\right)^{2}+\left(b^{*}\right)^{2}},
\end{array}
$$

where $\left(L_{t}^{*}, a_{t}^{*}, b_{t}^{*}\right)$ are a target color values (see Figure 1). Note that $L^{*}, a^{*}$, and $b^{*}$ are calculated according to surface spectral reflectance.

The calculated color difference shows how satisfactorily the predicted color matches the reference color. The use of $N N_{L a b}$ provides a way to take into account human visual sensitivity to color difference. Table 2 shows the performance of the color simulator $N N_{L a b}$. 


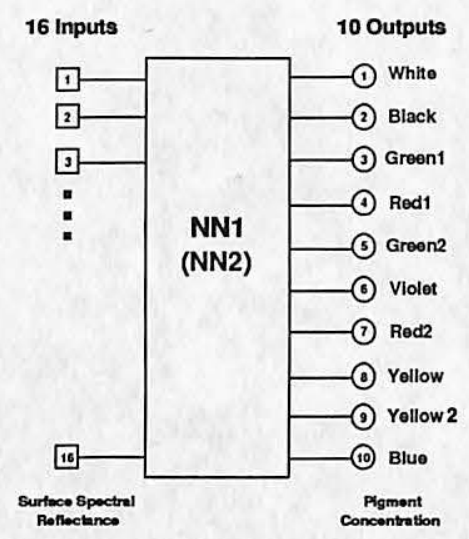

(A)

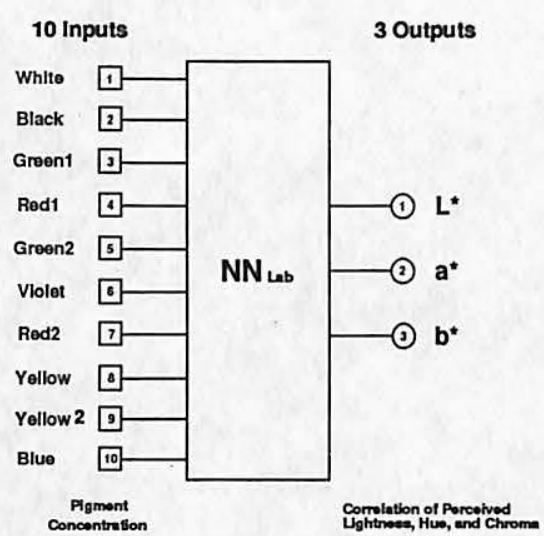

(B)

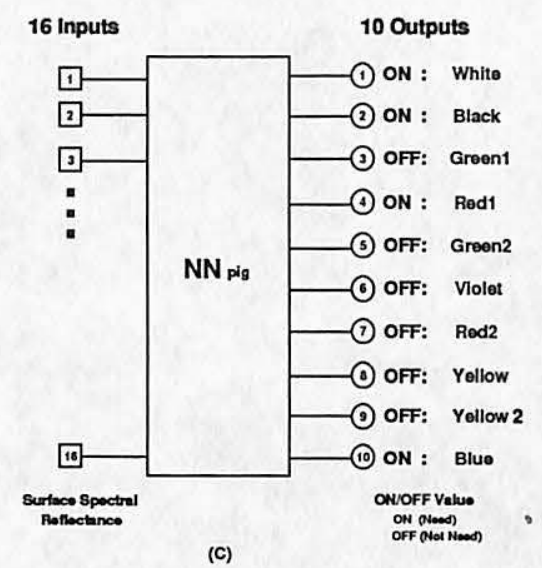

(C)

Fig. 3: Input/output relations of neural networks that are used in the proposed model.

\section{Genetic Strategies}

Genetic operations have a great impact on the quality of solutions. We have embodied some ideas in both mutation operations and crossover operations.

\subsection{Mutation Strategy}

Ordinary mutation operation as in a simple GA [3] is applied to all members with a fixed mutation rate scheme such that a fixed mutation rate $(0.01)$ is adopted with a probability of 0.4 , and otherwise, a mutation rate ranging from 0.09 to 0.69 , is decided using a random number. Moreover, the following modified operations are also considered.

\subsubsection{Chromosome Template}

To avoid specifying use of more pigments than necessary, we set out to inactivate some genes using the fuzzy population generator as described in Section 2.3. This has made it possible to use a chromosome itself as a template to do the mutation operation. Namely, before the mutation operation, it is decided whether to mutate an inactivated gene or not; the mutation is applied with low probability $(0.1)$ to inactivated genes, which have zero values of concentrations after decoding the genes' binary representations into pigment concentrations. If the mutation is applied to an inactivated gene, this leads to an increase in the number of necessary pigments.

\subsubsection{Local Search and Preservation of Multi-elites}

Multi-elites, i.e., chromosomes from the results of $\mathrm{NN}$ approaches and chromosomes originally generated by the multi-elite generator, are mutated only at the lower bits of each gene to keep traits similar to the NN results; Those mutant copies of the multielites can stay in the vicinity of the original multielites. In this way, local search of the NN results is realized. In addition, the offspring multi-elites always advance to the next generation; The mutant copies of multi-elites are preserved throughout the entire evolution. Note that this manipulation of low-order bits is applied only to the multi-elites.

\subsubsection{Exchanging Mutation}

After the usual mutation, members are subjected, with low probability, to another mutation: exchanging genes that have the same color information. This mutation is illustrated in Figure 4. Among the ten output pigment concentrations, we have three pairs of the same kinds of color pigments such as $\operatorname{Red}_{1}$ and $R e d_{2}$, which have actually different natures; we must decide which one to use. This exchanging mutation helps us to explore such color pigment choices. This can possibly lead to an escape from local optima in the initial $\mathrm{NN}$ and $N N_{p i g}$ results; their choices may not match the final choice determined by the system. The agreement with $N N_{p i g}$ in Table 4 shows how much the predicted choice optimized by the system matched the choices specified by $N N_{p i g}$.

\subsection{Modified Simplex Crossover}

We have modified the selection method of simplex crossover [1]. Our modified strategy uses the following three procedures:

(1) select one good chromosome with respect to fitness value using fitness proportionate selection; (2) select, with high probability, a multi-elite, as a good chromosome; and

(3) choose one bad chromosome with respect to fitness value.

This method may provide a better GA search direction as illustrated in Figure 5. When we have a 


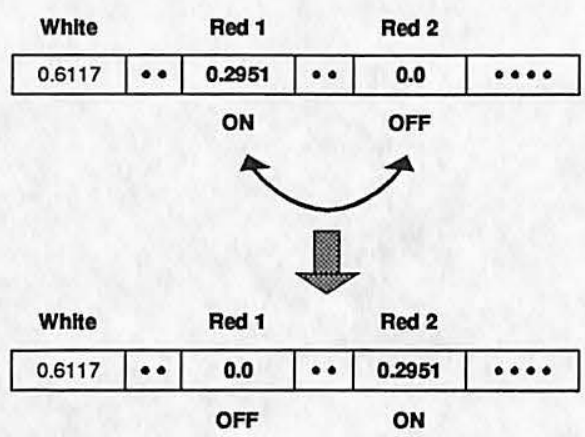

Fig. 4: Exchanging mutation

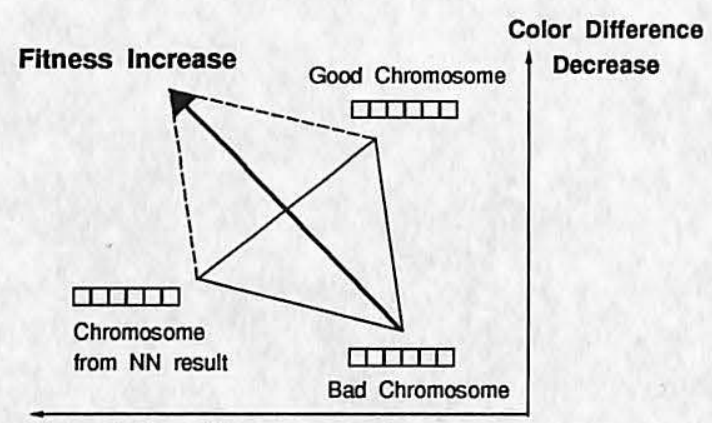

Pigment Error Decrease

Fig. 5: GA search control by modified simplex crossover.

neural fitness function, we may have a problem; a trained NN may not be a perfect fitness function. Indeed, both $N N_{p i g}$ and $N N_{L a b}$ in the fitness function are not perfect as shown in Table 1 and Table 2, but they may be able to direct a blind GA search to a better region of the search space. Procedure (1) lights a direction toward minimizing color difference. Thanks to $N N_{L a b}$, a chromosome with higher fitness may have smaller color difference. In this GA search, it is desirable to find a direction that minimizes both color difference and pigment errors. The problem is that we cannot calculate pigment errors directly. Yet the multi-elites provide a clue as to better pigment concentrations since they must already be within some range of the ideal pigment concentrations. That is why mutant copies from the multi-elites should be involved in guiding the paths towards better pigment concentration vectors as in procedure (2).

\section{Experimental Results}

The training data for NNs consist of 1,446 Munsell color (solid color) chips and the checking data of 111 standard paint color (solid color) chips of the Japan Paint Manufacturers Association. Both data sets are sampled from surface spectral reflectance at 16 points ranging from $400 \mathrm{~nm}$ to $700 \mathrm{~nm}$ in
Table: 3: Results of computational prediction in pigment error $\left(\times 10^{-2}\right)$ using 111 checking data.

\begin{tabular}{|c|c|c|c|}
\hline$N N_{1}$ & $N N_{2}$ & CNN & $G N F_{A L L}$ \\
\hline 2.312 & 1.543 & 1.139 & 0.643 \\
\hline
\end{tabular}

Table: 4: Performance evaluation in computational prediction of 111 checking data. $G N F_{A L L}$ shows the practical ability in the prediction task. A column, "agreement with $N N_{p i g}$ " implies how much the system match $N N_{\text {pig }}$ in specifying pigments; and "color diff. by $N N_{L a b}$ " shows the color difference predicted by $N N_{L a b}$.

\begin{tabular}{|c|c|c|c|c|}
\hline & $\begin{array}{c}\text { pigment } \\
\text { error } \\
\times 10^{-2}\end{array}$ & $\begin{array}{c}\text { ave. \# of } \\
\text { pigments } \\
\text { to use }\end{array}$ & $\begin{array}{c}\text { agreement } \\
\text { with } \\
N N_{\text {pig }}\end{array}$ & $\begin{array}{c}\text { color diff. } \\
\text { by } \\
N N_{L a b}\end{array}$ \\
\hline $\begin{array}{c}G N F_{A L L} \\
\text { (best) }\end{array}$ & 0.643 & 3.90 & $79.28 \%$ & 0.267 \\
\hline $\begin{array}{c}G N F_{A L L}^{\text {void }} \\
\text { (best) }\end{array}$ & 72.213 & 3.88 & $79.28 \%$ & 0.809 \\
\hline$G N F_{C P}$ & 1.190 & 3.94 & $50.45 \%$ & 48.800 \\
(best) & 0.206 & 4.02 & $21.62 \%$ & 34.637 \\
\hline$G N F_{C K}$ & 1.695 & 3.88 & $78.38 \%$ & 0.121 \\
(best) & 0.215 & 3.89 & $77.48 \%$ & 0.656 \\
\hline$G N F_{C}$ & 2.802 & 5.35 & $28.83 \%$ & 0.060 \\
(best) & 0.191 & 4.36 & $55.86 \%$ & 0.567 \\
\hline
\end{tabular}

wavelength (20-nm intervals). The configuration of the GA was as follows:

\section{Population size \\ Mutation rate \\ Crossover method \\ Simplex Crossover Rate \\ Generation \\ 80 members \\ flexible (see Section 3.1) \\ simplex crossover [1] \\ 0.85 \\ 10,000 .}

Table 3 shows the comparison of our proposed hybrid system, $G N F_{A L L}$, and some other approaches; $N N_{1}$ was a simple NN approach, and $\mathrm{NN}_{2}$ was an improved NN model that had modified sigmoidal functions in the output layer [8]. Both had the same model size $(16 \times 18 \times 21 \times 10$ neurons) (see Figure 3(A)). CNN was a neuro-fuzzy model [7]. $G N F_{A L L}$ employed all three results from $N N_{1}, N N_{2}$, and CNN in producing initial population. To exhibit how indispensable those $\mathrm{NN}$ results were, we examined $G N F_{A L L}^{\text {void }}$, which had no multielites but had the same conditions as GNF $F_{A L L}$.

Furthermore, to demonstrate the validity of each of three components in the fitness function, we tested $G N F_{C}, G N F_{C P}, G N F_{C K}$ to compare our proposed model, $G N F_{A L L}$. $G N F_{C}$ had $N N_{L a b}$ as the only component of the fitness function. $G N F_{C P}$ had both $N N_{L a b}$ and $N N_{p i g}$ as two components of the fitness function. $G N F_{C K}$ had a KB as well as $N N_{L a b}$ as two components.function. $G N F_{A L L}$ had all three components.

Note that $N N_{L a b}$ has an important role as a 
color simulator, and so it always must stay in the fitness function. Table 4 shows how each component contributed to the prediction, and how they complemented each other. In Table 4, (best) means the best performance with respect to pigment errors to show a potential capability of each model, regardless of fitness; they were obtained when pigment errors were minimized.

\section{Discussion}

Both $N N_{\text {pig }}$ and $N N_{L a b}$ as components of the fitness function made some prediction errors as shown in Tables 1 and 2. Thus, enhancing the overall performance may result from improving the accuracy of such neural fitness functions.

Basically, the main focus in recipe prediction should be color difference rather than pigment errors. Therefore, the system should work in conjunction with $N N_{L a b}$ to check predicted color difference. When $N N_{L a b}$ alone as the fitness function was employed in $G N F_{C}$, the system tended to go too far toward minimizing color difference, and therefore the average number of required pigments was larger. Additionally, the specified pigments did not match very well those designated by $N N_{p i g}$ as indicated in the low percentage of agreement with $N N_{p i g}$ in Table 4. $N N_{\text {pig }}$ was supposed to learn the characteristics of color pigment compositions such as complementary color relationships, yet it did not perform perfectly (see again Table 1). Thus, the KB surely helps the system evolve to recognize more features of color pigments. Here, it must be emphasized that they functioned complementarily.

$G N F_{A L L}^{v o i d}$ had no multi-elites but had all three components of the fitness function, starting from the randomly-initialized pigment concentration vectors. Its extraordinarily poor performance in predicted color difference places emphasis on the existence of the multi-elites, i.e., the mutant copies from other NN approaches; without them, we cannot draw any advantage from the search direction presented in Figure 5. In other words, seedings from other approaches are indispensable in enabling the system to function efficiently within a reasonable amount of computation time. Also, this poor performance may imply that fitness calculation should be altered for such no-seeding cases.

\section{Conclusions}

We have demonstrated both potential and practical strength of a unique blend of principal soft computing methods; a KB-based GA plays a leading role in pursuit of the prediction, linking an FS and NNs; they function complementarily as a system rather than competitively. The GA is not usually considered an ideal "fine-tuner" without specific modifi- cations; here we have discussed a fusion technique as well as the modifications applied to the fitness function and GA operations. The GA alone may not be a good fine-tuner, yet the complete resulting system can be viewed as a fine-tuner, overcoming individual limitations. The entire hybrid system has realized higher precision of prediction by evolving the results from other approaches

The proposed system will be an incredibly promising tool to reach our goal, and it would possibly revolutionize an approach to another industrial application.

\section{References}

[1] Bersini, H. and Seront, G., "In search of a good evolution-optimization crossover," Parallel Problem Solving from Nature, 2 R.Manner and B.Mandrick (editors), Elsevier Science Publishers, 1992, pp.479-488.

[2] Bishop, J.M., Bushnell, M.J., Westland, S., "Application of Neural Networks to Computer Recipe Prediction," Color Research and Application, Vol.16, No.1, pp.3-9, (1991)

[3] Goldberg, D., Genetic Algorithms in Search, Optimization, and Machine Learning, Addison-Wesley (1989)

[4] Jang, J.-S. Roger, Sun, C.-T., and Mizutani, E., Neuro-Fuzzy Modeling and Soft Computing, Prentice Hall (in press)

[5] Kido, T., Kitano, H., Nakanishi, M., "A Hybrid Search for Genetic Algorithms: Combining Genetic Algorithms, TABU Search, and Simulated Annealing," 5th Int'l Conf. on Genetic Algorithms (ICGA'93), p.640 (July, 1993)

[6] Mansour, N., Fox, G.C., "A Hybrid Genetic Algorithm for Task Allocation in Multicomputers," 4th Int'l Conf. on Genetic Algorithms (ICGA'91), pp.466-473 (July, 1991)

[7] Mizutani, E., Jang, J-S. Roger., Nishio, K., Takagi, H., Auslander, D.M., "Coactive Neural Networks with Adjustable Fuzzy Membership Functions and Their Applications," Int'l Conf. on Fuzzy Logic, Neural Nets, and Soft Computing (IIZUKA '94), pp.581-582 (Aug., 1994)

[8] Mizutani, E., Takagi, H., Auslander, D.M., "A Cooperative system based on soft computing methods to realize higher precision of computer color recipe prediction," Applications and Science of Artificial Neural Networks, part of SPIE's Int'l Symposium on OE/Aerospace Sensing and Dual Use Photonics (April, 1995)

[9] Spehl, J., Wolker, M., Pelzl, J., "Application of Backpropagation Nets for Color Recipe Prediction as a Nonlinear Approximation Problem," IEEE Int'l Conf. on NN (ICNN'94), pp.3336-3341 (June, 1994)

[10] Wyszecki, G. and Stiles, W.S., Color science: concepts and methods, quantitative data and formulae, New York, NY: Wiley, 2nd ed. (1982) 